\title{
Doenças Cardiovasculares antes e após o Programa Saúde da Família, Londrina, Paraná
}

\author{
Diseases of the Circulatory System before and after the Family Health Program, Londrina, Paraná \\ Brígida Gimenez Carvalho, Regina Kazue Tanno de Souza, Darli Antonio Soares, Mara Cristina Nishikawa Yagi \\ Departamento de Saúde Coletiva da Universidade Estadual de Londrina, PR - Brasil
}

\section{Resumo}

Fundamento: A redução da morbi-mortalidade pelas doenças do aparelho circulatório (DAC) é um dos maiores desafios da atenção básica, e a atuação da Saúde da Família possibilita o acesso às medidas multissetoriais e integrais que a abordagem dessas doenças exige.

Objetivo: Analisar a mortalidade e a internação hospitalar, por DAC, antes e após a implantação da Saúde da Família em Londrina-PR.

Métodos: Estudo de agregados, comparando-se os coeficientes de mortalidade e de internação hospitalar pelo SUS, por DAC, doença cerebrovascular (DCbV) e doença isquêmica do coração (DIC), de residentes em Londrina, em dois quadriênios: 1997 a 2000 e 2002 a 2005. Os dados foram obtidos no Sistema de Informações sobre Mortalidade e no Sistema de Informações Hospitalares do SUS. Foram calculadas razões entre as taxas nos dois períodos e os respectivos intervalos de $95 \%$ de confiança.

Resultados: As DAC mantiveram-se como a primeira causa de morte no município nos dois quadriênios. As DCbV e as DIC responderam por mais de $63 \%$ das mortes por DAC. Na comparação dos dois quadriênios, observou-se a redução das taxas de mortalidade por DCbV, significativa apenas em maiores de 59 anos, em ambos os sexos, e o aumento da internação em 10\%. Para as DIC não houve alteração significativa na mortalidade e ocorreu um aumento de $40 \%$ na taxa de internação.

Conclusão: A redução significativa apenas na mortalidade por DCbV em idosos sugere a necessidade de ampliar a cobertura assistencial aos diferentes grupos populacionais e o desenvolvimento de ações de caráter preventivo e de promoção à saúde. (Arq Bras Cardiol 2009; 93(6):

$-650)$

Palavras-chave: doenças cardiovasculares/mortalidade, morbidade, avaliação de programas e projetos de saúde.

\section{Summary}

Background: The reduction in morbidity and mortality rates from diseases of the circulatory system (DCS) is one of the greatest challenges in primary care, and the implementation of the Family Health Program provides access to the multisectoral and integrated measures required for dealing with these diseases.

Objective: To analyze DCS mortality and hospitalization rates before and after the implementation of the Family Health Program, in Londrina, Paraná.

Methods: This was an ecological study, comparing DCS, cerebrovascular disease (CbVD) and ischemic heart disease (IHD) mortality rates and hospitalization rates by the SUS, in residents of Londrina, in two 4-year periods: 1997 to 2000, and 2002 to 2005. The data were obtained from the Mortality Information System and the Hospital Information System of the SUS. The ratios between the rates were calculated for both periods at $95 \%$ confidence interval.

Results: The DCS was the first cause of death in the city, in both quadrennia. The CbVD and IHD accounted for more than 63\% of DCS deaths. In comparing the two quadrennia, there was a reduction in CbVD mortality rates, which was significant only for subjects over 59 years, in both genders, and the hospitalization rates increased by $10 \%$. For IHD, no significant change occurred in mortality rates, and there was a $40 \%$ increase in hospitalization rates.

Conclusion: The significant reduction only in CbVD mortality in the elderly suggests the need for expanding health assistance coverage to different population groups and the development of preventive and health promotion measures. (Arq Bras Cardiol 2009; 93(6):597-601)

Key words: diseases/mortality; morbidity; program evaluation.

Full texts in English - http://www.arquivosonline.com.br

Correspondência: Brígida Gimenez Carvalho •

Rua Chile, 43 - 10 andar - Centro - 86010-220 - Londrina- PR - Brasil

E-mail: brigidagimenez@gmail.com

Artigo recebido em 15/07/2008; revisado recebido em 16/10/2008; aceito em 10/11/2008. 


\section{Introdução}

A revolução tecnológica e industrial produziu mudanças no perfil de morbi-mortalidade da população, com predomínio das doenças e mortes provocadas pelas doenças crônicas não transmissíveis (DCNT), que produzem elevados custos para a sociedade, devido à alta mortalidade e invalidez precoces ${ }^{1}$.

Na América Latina e no Caribe, as DCNT constituem a principal causa de morte, e as de maior importância são as doenças do aparelho circulatório (DAC), os cânceres e o diabetes mellitus ${ }^{2}$. No Brasil, as DAC foram responsáveis, no ano de 2005, por 31,5\% dos óbitos. Entre essas, a doença cerebrovascular (DCbV) foi a primeira causa de morte, seguida pelo infarto agudo do miocárdio, que é a principal dentre o grupo das doenças isquêmicas do coração (DIC). No ano de 2006, as DAC representaram 10,1\% do total de internações ${ }^{3}$.

Tanto as DCbV como as DIC têm fatores de risco bem estabelecidos. O estudo de Framingham foi um dos primeiros a demonstrar que a hipertensão arterial, altos níveis de colesterol, obesidade, diabetes mellitus, falta de atividade física e tabagismo são fatores de risco fortemente relacionados ao desenvolvimento da doença cardíaca e cerebrovascular ${ }^{4}$. Após esse estudo, vários outros estudos confirmaram essa relação $\mathrm{O}^{5-7}$.

Todos esses fatores de risco estão de alguma forma relacionados, portanto, é de fundamental importância a implementação de modelos de atenção à saúde que incorporem estratégias diversas de prevenção e/ou controle desses fatores, individuais e coletivos, a fim de contribuir para a redução da morbi-mortalidade pelas DAC. Essa é, na atualidade, um dos maiores desafios da atenção básica ${ }^{8}$.

A adoção da estratégia Saúde da Família como política prioritária de atenção básica, por sua conformação e processo de trabalho, compreende as condições mais favoráveis de acesso às medidas multissetoriais e integrais que a abordagem das DAC exige ${ }^{1}$.

Diante da relevância das DAC no quadro nosológico no país e no mundo, e considerando a atuação das equipes de saúde da família na identificação e no controle dos principais fatores de risco das DAC, é objetivo deste estudo analisar os coeficientes de mortalidade e de internação hospitalar pelo SUS, antes e após a implantação da Saúde da Família no município de Londrina - PR.

\section{Métodos}

Trata-se de um estudo de agregados, no qual foram comparados coeficientes de mortalidade e de internações hospitalares por DAC, DCbV e DIC, de pessoas residentes no município de Londrina, em dois quadriênios: 1997 a 2000 e 2002 a 2005, respectivamente antes e após a implantação da estratégia Saúde da Família. O ano de 2001 foi excluído por corresponder ao ano da implantação dessa estratégia na área urbana do município.

O município de Londrina situa-se na região norte do Estado do Paraná e contava, no ano de 2005, com uma população de 488287 habitantes ${ }^{9}$. Possuía, nesse mesmo ano, uma rede de atenção básica composta por 54 unidades de saúde, nas quais se encontravam vinculadas 102 equipes de Saúde da Família, com uma cobertura de mais de $70 \%$ da população do município, principalmente a de áreas periféricas e de maior risco epidemiológico e social ${ }^{10}$.

Os dados de mortalidade e de internação hospitalar foram obtidos no banco do Sistema de Informações sobre Mortalidade (SIM) e do Sistema de Informações Hospitalares do Sistema Único de Saúde (SIH/SUS). Esses bancos são disponibilizados pelo Departamento de Informática do SUS (DATASUS), órgão sob responsabilidade da Secretaria Executiva do Ministério da Saúde ${ }^{11}$. As informações sobre a população tiveram como fonte o Instituto Brasileiro de Geografia e Estatística (IBGE), contagem populacional de 1996 e estimativas para 1997 a 1999 e o Censo de 2000 (primeiro quadriênio) e estimativas do Censo de 2000 para 2002 a 2005 (segundo quadriênio) ${ }^{9}$.

A coleta e classificação dos dados pelos Sistemas de Informações do Ministério da Saúde sobre a morbidade e mortalidade por doenças do aparelho circulatório baseouse na classificação estatística internacional de doenças e problemas relacionados à saúde, X Revisão da Classificação Internacional de Doenças (CID-10), capítulo IX ${ }^{12}$. Para obtenção do número de internações no ano de 1997, foi utilizado a CID - 9 (capítulo VII) que era a classificação em vigor naquele período. As DAC foram agrupadas pelos códigos: CID-9 390 a 459 e CID-10 I00 a 199; as DCbV agrupadas pelos códigos: CID-9 430 a 438 e CID-10 I60 a 169; e as DIC agrupadas pelos códigos: CID-9 410 a 414 e CID-10 I20 a I25.

Os dados foram transferidos para a planilha eletrônica Excel e em seguida foram construídas taxas específicas, segundo as variáveis: sexo, faixa etária, causa de morte e de internação hospitalar. Foram utilizados os coeficientes brutos de mortalidade e de internação hospitalar por DAC, DCbV e DIC, em vez dos padronizados, considerando-se a semelhança da estrutura populacional do município nos dois períodos. Segundo Laurenti e cols. ${ }^{13}$, a padronização constitui recurso a ser utilizado na comparação de populações com diferentes estruturas etárias.

Para comparação das taxas antes e após a implantação da Saúde da Família, foram calculadas razões de mortalidade e de internação hospitalar e os respectivos intervalos de 95\% de confiança para as causas estudadas, tendo por referência o valor correspondente ao quadriênio 1997-2000. Para o cálculo do intervalo de confiança, utilizou-se o "statcalc" do Programa Epi Info, Versão 3.4.1. Considerando-se a estratégia Saúde da Família como intervenção, razões inferiores à unidade, inclusive no intervalo de confiança, expressariam redução estatisticamente significativa nas taxas de ocorrência dos agravos no segundo período.

O projeto de pesquisa foi submetido ao Comitê de Ética em Pesquisa envolvendo Seres Humanos da Universidade Estadual de Londrina, sendo analisado e aprovado sob o parecer 201/06 em 07 de agosto de 2006.

\section{Resultados}

As DAC mantiveram-se como a primeira causa de morte no município de Londrina nos dois quadriênios estudados, 
respondendo por 3262 mortes no primeiro quadriênio e por 3389 no segundo. Dentre as DAC, as DCbV foram as mais prevalentes (40,31\% das DAC no primeiro quadriênio e $37 \%$ no segundo), seguidas pelas DIC (25,1\% e $26,82 \%$, respectivamente). As DCbV e as DIC responderam juntas, em ambos os períodos, por aproximadamente $65 \%$ das mortes por DAC. Apesar da redução do coeficiente de mortalidade por DAC no segundo período, que passou de 188,9 para $179,3 / 100.000$ habitantes, esta não foi significativa (razão $=$ 0,95, I.C.95\%: 0,90-1,00).

Quanto às internações hospitalares por DAC, o coeficiente de internação hospitalar manteve-se inalterado nos dois períodos (razão = 1,00, I.C.95\%: 0,97: 1,02). Já as DCbV foram responsáveis por 9,6\% e 10,7\% do total de internações por DAC, no primeiro e segundo período respectivamente, mas a participação percentual das DIC aumentou expressivamente no segundo período, passando de $19,7 \%$ para $27,1 \%$.

A tabela 1 apresenta os coeficientes de mortalidade e de internação hospitalar das DCbV. Observa-se uma redução das taxas de mortalidade (razão= 0,87, I.C.95\%: 0,81-0,94), mas um aumento de $10 \%$ no coeficiente de internação no segundo período (razão=1,10, I.C.95\%: 1,03-1,19), sendo ambos estatisticamente significativos.

Tabela 1 - Coeficientes de mortalidade e internação hospitalar pelo SUS por doença cerebrovasculares (por 100.000 hab.) segundo sexo e grupo etário. Londrina - PR, 1997-2000 e 2002-2005

\begin{tabular}{|c|c|c|c|c|}
\hline Coeficiente & & $1997-2000$ & $2002-2005$ & $\begin{array}{c}\text { Razão } \\
\text { 2002- 2005/ } \\
\text { 1997-2000 } \\
\text { IC (95\%) }\end{array}$ \\
\hline Mortalidade & & 76,1 & 66,3 & $0,87[0,81: 0,94]^{*}$ \\
\hline \multirow{4}{*}{ Masculino } & & 87,5 & 77,0 & $0,88[0,79: 0,98]^{\star}$ \\
\hline & 30 a 44 & 15,6 & 15,2 & $0,97[0,59: 1,62]$ \\
\hline & 45 a 59 & 82,1 & 94,9 & $1,16[0,88: 1,52]$ \\
\hline & $60 \mathrm{e}+$ & 886,0 & 682,0 & $0,77[0,69: 0,86]^{*}$ \\
\hline \multirow{4}{*}{ Feminino } & & 65,5 & 56,4 & $0,86[0,77: 0,97]^{*}$ \\
\hline & 30 a 44 & 10,9 & 7,3 & $0,67[0,36: 1,26]$ \\
\hline & 45 a 59 & 46,4 & 45,8 & $0,99[0,69: 1,41]$ \\
\hline & $60 \mathrm{e}+$ & 619,6 & 475,5 & $0,77[0,68: 0,87]^{\star}$ \\
\hline Internação & & 78,1 & 86,7 & $1,10[1,03: 1,19]^{*}$ \\
\hline \multirow{4}{*}{ Masculino } & & 85,7 & 106,7 & $1,25[1,13: 1,37]^{*}$ \\
\hline & 30 a 44 & 37,2 & 43,2 & $1,16[0,85: 1,59]$ \\
\hline & 45 a 59 & 169,8 & 203,2 & $1,20[0,99: 1,45]$ \\
\hline & $60 \mathrm{e}+$ & 635,6 & 719,5 & $1,13[1,00: 1,28]^{*}$ \\
\hline \multirow{4}{*}{ Feminino } & & 70,9 & 66,9 & $0,73[0,85: 1,05]$ \\
\hline & 30 a 44 & 31,2 & 22,8 & $0,73[0,51: 1,05]$ \\
\hline & 45 a 59 & 109,8 & 100,5 & $0,91[0,72: 1,16]$ \\
\hline & $60 e+$ & 488,0 & 437,2 & $0,90[0,78: 1,03]$ \\
\hline
\end{tabular}

*Diferença estatisticamente significativa.
Ao estratificar por sexo, verifica-se uma redução significativa no coeficiente de mortalidade, tanto para os homens quanto para as mulheres, porém, na estratificação por faixa etária, essa redução foi significativa apenas no grupo de maiores de 60 anos, em ambos os sexos.

Para o coeficiente de internação hospitalar por DCbV, observou-se um aumento significativo do coeficiente entre os homens. Nas mulheres, verificou-se, apesar de não significativa, uma redução em todos os grupos etários. Os coeficientes de mortalidade e de internação hospitalar por DCbV também foram mais elevados no sexo masculino. A relação masculino/feminino manteve-se relativamente estável para a mortalidade, passando de 1,3 no primeiro quadriênio para 1,4 no segundo. Para a internação, houve um aumento expressivo na relação masculino/feminino, passando de 1,2 para 1,6 no segundo quadriênio.

A tabela 2 mostra que, relativamente às DIC, não houve alteração significativa no coeficiente de mortalidade entre os períodos (razão=1,01, I.C.95\%: 0,92-1,12). Alterações discretas e não significativas foram observadas nas taxas de mortalidade específicas por sexo. Entre os homens houve um pequeno aumento, sobretudo no grupo mais jovem (30 a 45 anos) e entre as mulheres, redução em todos os grupos etários.

Tabela 2 - Coeficientes de mortalidade e de internação hospitalar pelo SUS por doenças isquêmicas do coração (DIC) (por 100.000 hab.), segundo sexo e grupo etário. Londrina - PR, 1997-2000 e 2002-2005

\begin{tabular}{|c|c|c|c|c|}
\hline Coeficiente & & $1997-2000$ & $2002-2005$ & $\begin{array}{c}\text { Razão } \\
\text { 2002- 2005/ } \\
\text { 1997-2000 } \\
\text { IC (95\%) }\end{array}$ \\
\hline Mortalidade & & 47,4 & 48,1 & $1,01[0,92: 1,12]$ \\
\hline \multirow{4}{*}{ Masculino } & & 57,2 & 60,7 & $1,06[0,94: 1,20]$ \\
\hline & 30 a 44 & 18,9 & 23,1 & $1,22[0,79: 1,90]$ \\
\hline & 45 a 59 & 96,7 & 95,7 & $0,99[0,76: 1,28]$ \\
\hline & $60 \mathrm{e}+$ & 486,2 & 474,3 & $0,98[0,84: 1,13]$ \\
\hline \multirow{4}{*}{ Feminino } & & 38,3 & 36,3 & $0,95[0,82: 1,10]$ \\
\hline & 30 a 44 & 5,2 & 3,0 & $0,58[0,22: 1,49]$ \\
\hline & 45 a 59 & 48,0 & 32,8 & $0,68[0,47: 1,00]$ \\
\hline & $60 \mathrm{e}+$ & 334,0 & 308,0 & $0,92[0,78: 1,09]$ \\
\hline Internação & & 160,6 & 220,6 & $1,40[1,31: 1,44]^{*}$ \\
\hline \multirow{4}{*}{ Masculino } & & 195,5 & 257,2 & $1,32[1,24: 1,40]^{*}$ \\
\hline & 30 a 44 & 88,9 & 100,7 & $1,13[0,92: 1,39]$ \\
\hline & 45 a 59 & 509,3 & 685,0 & $1,35[1,21: 1,50]^{*}$ \\
\hline & $60 \mathrm{e}+$ & 1325,4 & 1549,2 & $1,17[1,07: 1,27]^{*}$ \\
\hline \multirow{4}{*}{ Feminino } & & 127,4 & 186,1 & $1,46[1,36: 1,58]^{*}$ \\
\hline & 30 a 44 & 35,5 & 38,3 & $1,08[0,79: 1,47]$ \\
\hline & 45 a 59 & 296,1 & 367,6 & $1,24[1,09: 1,42]^{*}$ \\
\hline & $60 e+$ & 851,8 & 1223,9 & $1,44[1,31: 1,58]^{*}$ \\
\hline
\end{tabular}

*Diferença estatisticamente significativa. 
Em relação ao coeficiente de internação hospitalar, houve um aumento significativo no segundo quadriênio, com acréscimo de 40\% na taxa (razão=1,40, I.C.95\%: 1,31$1,44)$. Essa elevação se deu de maneira homogênea nos dois sexos e em todos os grupos. Porém, foi nas faixas etárias de 45 a 59 anos e nos maiores de 60 anos que o aumento foi estatisticamente significativo.

Também para as DIC, os coeficientes de mortalidade e de internação hospitalar foram superiores no sexo masculino. Para o coeficiente de mortalidade, a relação masculino/feminino que era de 1,5 no primeiro quadriênio passou para 1,7 no segundo. Já para o coeficiente de internação hospitalar, a relação masculino/ feminino apresentou redução no segundo quadriênio em relação ao primeiro, passando de 1,5 para 1,4.

\section{Discussão}

Quanto ao delineamento dos estudos ecológicos ou de agregados, segundo Almeida Filho e Rouquayrol ${ }^{14}$, só recentemente, inicia-se uma reavaliação das bases lógicas e metodológicas desse tipo de estudo, tendo pela primeira vez merecido o destaque de um capítulo específico no mais importante livro texto de epidemiologia da atualidade. No âmbito da epidemiologia, os estudos ecológicos passaram por um período de intenso processo de desvalorização, sendo relegados à condição de abordagem meramente descritiva, sem maior poder analítico.

Segundo Morgenstern ${ }^{15}$, os efeitos ecológicos são particularmente relevantes quando se tem por objetivo avaliar intervenções na população, tais como novo programa, políticas ou legislação. Todavia, algumas das limitações citadas por vários autore ${ }^{15-17}$ merecem ser consideradas na análise dos resultados do presente estudo. A primeira refere-se à qualidade da informação e a segunda à dificuldade de controlar fatores de confundimento.

No tocante às informações utilizadas, os dados do município de Londrina, com baixas proporções de causas mal definidas - observadas após a implantação do Núcleo de Informações em Mortalidade (NIM), em meados da década de 1990, que checa as declarações de óbito e realiza investigação para confirmar a verdadeira causa de óbito ${ }^{18}$-, sugerem a boa qualidade das informações. Merece destaque o fato das causas mal definidas representarem, no período estudado, menos de $1,7 \%$ das mortes ${ }^{19}$.

As diferenças significativas encontradas nos valores dos coeficientes analisados não podem ser entendidas exclusivamente como resultantes da intervenção (PSF), pois não foi de alcance do estudo o controle dos fatores de confundimento. Portanto, apenas permitem aventar hipóteses sobre uma possível influência da estratégia de atenção implantada nos níveis de morbi-mortalidade por doenças cardiovasculares, que deve ser mais bem explorada a partir do uso de outros delineamentos como, por exemplo, estudo de caso controle.

Quanto aos resultados, este estudo identificou uma redução de $5,1 \%$ no coeficiente de mortalidade por DAC no período de 2002 a 2005 em relação ao período de 1997 a 2000. O grupo de causas responsável por essa redução foi o das DCbV, com redução de 12,9\% no coeficiente. Esses resultados acompanham a tendência de queda das DAC e DCbV que vem ocorrendo na região Sul do país ${ }^{20}$ e em alguns países como Japão, Austrália, Estados Unidos e países da Europa Ocidental, desde a década de $1950^{21}$. As taxas de mortalidade por DCbV apresentadas pelo município no segundo período foram mais baixas que as da região Sul do país em 2001, nos dois sexos e em todos os grupos etários ${ }^{22}$.

Vários estudos têm demonstrado que as DCbV apresentam aumento significativo da mortalidade com o aumento da idade, atingindo valores extremamente altos em pessoas acima dos 60 anos $^{22-24}$, situação também confirmada por este estudo no município de Londrina. A redução significativa na taxa de mortalidade DCbV leva a inferir que a atenção prestada pela Saúde da Família no município, que tem a hipertensão arterial como área programática prioritária para atuação das equipes (por meio da dispensação de medicamentos, controle da pressão arterial, acompanhamento clinico e laboratorial dos doentes) tem contribuído para a redução dos coeficientes de mortalidade, entre mulheres e idosos. Ressalta-se que as ações de controle da hipertensão arterial pelas equipes de Saúde da Família atingem principalmente estes dois grupos: mulheres e idosos, conforme descrito por Sousa e cols. ${ }^{25}$ na atuação dessa estratégia em município de pequeno porte do interior do Paraná.

No estudo realizado por Abreu e cols. ${ }^{26}$, verificou-se o declínio da mortalidade pela DCbV no Brasil, no período de 1993 a 2002. Os autores atribuíram esta redução, em parte, às mudanças na oferta e no acesso aos serviços de saúde ocorridos no país nesse período. Conforme estudo conduzido por Collins e cols. ${ }^{27}$, a DCbV é um agravo que pode ser evitado em até $42 \%$ com controle adequado da hipertensão arterial sistêmica. No entanto, também deve se considerar que a facilidade de acesso às internações hospitalares pode ter contribuído para reduzir a letalidade e, portanto, a mortalidade por essa causa.

No caso das DIC, o comportamento da doença no município, com semelhanças nos valores assumidos pelos coeficientes nos dois períodos estudados, difere da tendência à queda verificada nos Estados Unidos, países da Europa Ocidental, Japão e também na América Latina desde o final dos anos 1960, e nas regiões Sul e Sudeste do Brasil desde OS anos $1980^{28}$. Segundo Lotufo ${ }^{28}$, a mortalidade pela DIC no Brasil não é uniforme. Quanto às internações hospitalares em Londrina, o aumento nas taxas pode ser consequente à ampliação no acesso à atenção hospitalar, reflexo da regulação da atenção pré-hospitalar e hospitalar, com a implantação do Serviço de Atendimento Móvel de Urgência (SAMU) e da central de leitos no município no início da década de 2000. Outra questão a ser considerada no aumento mencionado, refere-se ao avanço tecnológico no diagnóstico e tratamento intervencionista para esse agravo (revascularização do miocárdio e angioplastias) verificado nos últimos anos. Segundo Oliveira e cols. ${ }^{29}$, em um estudo realizado no Estado do Rio de Janeiro, houve um aumento significativo das internações por DIC, com crescimento concomitante de procedimentos de alta complexidade nessas internações.

Apesar do aumento da internação, não houve redução da mortalidade por DIC. A esse respeito, há que se destacar que não há consenso sobre qual tipo de intervenção médica 
tem maior impacto na ocorrência dessa doença ${ }^{26}$. No estudo "INTERHEART", realizado em 52 países, avaliando o efeito de fatores de risco para o infarto do miocárdio, verificou que as dislipidemias; o tabagismo; a hipertensão; o diabetes; a obesidade abdominal; o baixo consumo diário de frutas e verduras; o uso de álcool; e a falta de atividade física regular são os mais importantes fatores de risco em todos os países, em ambos os sexos e em todas as idades. Esses nove fatores responderam por $90 \%$ do risco atribuível nos homens e $94 \%$ nas mulheres ${ }^{30}$.

Essa constatação reforça o caráter multifatorial das doenças cardiovasculares e a insuficiência das ações intervencionistas, e daquelas que focam prioritariamente o controle da hipertensão arterial em idades mais avançadas. Há que se considerar que na etiologia da DIC, o adequado controle da hipertensão arterial sistêmica (HAS), pode diminuir a ocorrência da DIC em $14 \%{ }^{27}$. Tais aspectos indicam a necessidade de redirecionamento da atenção e ampliação da cobertura assistencial, com abordagens que contemplem mudanças no estilo de vida, de caráter preventivo e de promoção à saúde.

Cabe a reflexão sobre que estratégias preventivas e assistenciais deveriam ser adotadas pelas equipes de Saúde da Família, visando a redução das mortes por DIC e por DCbV nos outros grupos etários e principalmente na população masculina. Nesse sentido, seria fundamental que as várias iniciativas para reduzir o impacto das doenças não-transmissíveis na população brasileira, propostas pelo Ministério da Saúde, que incluem o rastreamento do diabetes no nível nacional, a implementação de campanhas sobre hipertensão arterial sistêmica e a aplicação de protocolos para manejo agressivo da dislipidemia em coronariopatas ${ }^{31}$, também encontrem condições adequadas para o seu desenvolvimento pelas equipes locais.

Destaque-se que só mais recentemente estão sendo adotadas estratégias de promoção à saúde. A Política Nacional de Promoção à Saúde, publicada em 2002 pelo Ministério da Saúde ${ }^{32}$, fez vários apontamentos, porém não foi suficiente para deflagrar o desenvolvimento de ações concretas nesse campo. Uma estratégia recente, divulgada no início de 2008 por meio de portaria ministerial, foram os Núcleos de Apoio à Saúde da Família ${ }^{33}$, de atuação multiprofissional, com objetivo de apoiar as equipes de saúde da família e que, se bem sucedidos, constituirão um importante passo para o desenvolvimento de ações de atividade física, de promoção de práticas alimentares saudáveis, de saúde mental, dentre outras, reconhecidamente necessárias ao enfrentamento desses agravos não transmissíveis.

Apesar de a presente análise basear-se em dados secundários e agregados, os resultados mostraram a redução das taxas de mortalidade por doenças do aparelho circulatório no município, após a implantação da estratégia Saúde da Família, como consequência da diminuição da mortalidade por doenças cerebrovasculares, notadamente na população idosa. Os resultados indicam, de certo modo, o impacto dos investimentos no controle da hipertensão arterial e na prevenção dos eventos cerebrovasculares. Todavia, a manutenção dos níveis de mortalidade e o incremento de internações hospitalares, tanto por DCbV quanto por DIC, entre adultos não idosos, apontam a necessidade de se aprofundar a análise em nível local, no sentido de verificar em que medida as ações têm alcançado diferentes grupos populacionais, em especial os inseridos no mercado de trabalho. Além disso, é também merecedora de preocupação a sobremorbi-mortalidade masculina observada, principalmente ao se considerar que avanços ulteriores na promoção da saúde cardiovascular dependem, em grande parte, da capacidade de inclusão das pessoas tradicionalmente à margem do sistema de saúde, com destaque para a atenção básica. É necessário que os indicadores epidemiológicos não sejam calculados apenas como resposta às exigências governamentais para o repasse de recursos, mas que sejam incorporados ao conceito de vigilância e de acompanhamento sistematizado, com o propósito de dar suporte aos processos decisórios em toda linha do cuidado.

\section{Potencial Conflito de Interesses}

Declaro não haver conflito de interesses pertinentes.

\section{Fontes de Financiamento}

O presente estudo não teve fontes de financiamento externas.

\section{Vinculação Acadêmica}

Este artigo é parte da monografia de especialização em Saúde Coletiva de Mara Cristina Nishikawa Yagi pela Universidade Estadual de Londrina.

\section{Referências}

1. Ministério da Saúde. Secretaria de Atenção à Saúde. Departamento de Atenção Básica. Prevenção clínica de doença cardiovascular, cerebrovascular e renal crônica. Brasília; 2006. (Cadernos de Atenção Básica, 14).

2. Malta DC, Morais Neto OL. Introdução. In: Ministério da Saúde. Secretaria de Vigilância em Saúde. Departamento de Análise de Situação de Saúde. Guia metodológico de avaliação e definição de indicadores: doenças crônicas não transmissíveis e Rede Carmen. Brasília; 2007. p. $18-20$.
3. Ministério da Saúde [homepage na internet]. Caderno de Informações de Saúde, 2007. [acesso em 2007 Set 9]. Jul. Disponível em: http://tabnet. datasus.gov.br/tabdata/cadernos/BR/Brasil GeralBR.xls.

4. Sytkowsly PA, Kannel WB, D'Agostino RB. Changes in risk factors and the decline in mortality from cardiovascular disease. The Framingham heart Study. N Engl J Med. 1990; 322: 1635-41.

5. Lewington S, Clarke R, Qizilbash N, Peto R, Collins R, Prospective Studies 
Collaboration. Age-specific relevance of usual blood pressure to vascular mortality: a meta-analysis of individual data for one million adults in 61 prospective studies. Lancet. 2002; 360: 1903-13.

6. Yusuf S, Hawken S, Ounpuu S, Dans T, Avezum A, Lanas F, et al. Effect of potentially modifiable risk factors associated with myocardial infarction in 52 countries (the INTERHEART study): case-control study. Lancet. 2004; 364: 937-52.

7. Avezum A, Piegas LS, Pereira JC. Fatores de risco associados com infarto agudo do miocárdio na região metropolitana de São Paulo: uma região desenvolvida em um país em desenvolvimento. Arq Bras Cardiol. 2005; 84(3): 206-13.

8. Ministério da Saúde. Secretaria de Atenção à Saúde. Departamento de Atenção Básica. Hipertensão arterial sistêmica para o Sistema Único de Saúde. Brasília; 2006. (Cadernos de Atenção Básica, 16).

9. Instituto Brasileiro de Geografia e Estatística. (IBGE). [homepage na internet]. População [acesso em 2007 Ago 16]. Disponível em: http://www.ibge.gov. br/home/estatistica/populacao/estimativa2005/estimativa.shtm.

10. Prefeitura do Município de Londrina. Secretaria Municipal de Saúde. Plano Municipal de Saúde 2006-2007. Londrina; 2007.

11. Ministério da Saúde [homepage na internet]. DATASUS. Informações de saúde. Morbidade e informações epidemiológicas. [Acesso em 2009 abr 18]. Disponível em: http://w3.datasus.gov.br

12. Organização Mundial da Saúde. Classificação estatística internacional de doenças e problemas relacionados à saúde. 10ª ed. São Paulo: EDUSP; 1995.

13. Laurenti R, Melo Jorge MHP, Lebrão MI, Gotlieb SLD. Estatísticas de saúde. $2^{\text {a }}$ ed. São Paulo: EPU; 2005.

14. Almeida Filho M, Rouquayrol MZ. Elementos da metodologia epidemiológica. In: Rouquayrol MZ, Almeida Filho M. (eds). Epidemiologia e saúde. $6^{\underline{a}}$ ed. Rio de Janeiro: MEDSI; 2003. p. 149-77.

15. Morgenstern H. Ecologic studies. In: Rothman KJ, Greenland S. Modern epidemiology. Philadelphia: Lippincott Williams \& Wilkins; 1998. p. 459-80.

16. Pereira MG. Epidemiologia teoria e prática. Rio de janeiro: Editora Guanabara Koogan; 1995. p. 269-88.

17. Medronho RA. Estudos ecológicos. In: Medronho RA, Carvalho DM, Bloch KV, Luiz RR, Werneck GL. Epidemiologia. São Paulo: Editora Atheneu; 2005. p. 191-8.

18. Andrade SM, Soares DA. Dados e informação em saúde: para que servem? In: Andrade SM, Soares DA, Cordoni Junior L, (orgs.). Bases da saúde coletiva. Londrina: Ed. UEL; 2001. p. 161-81.

19. Ministério da Saúde [homepage na internet]. DATASUS. Informações de saúde. [Acesso em 2008 out 13]. Disponível em: http://tabnet.datasus.gov.br

20. Mansur AP, Souza MFM, Timerman A, Avakian SD, Aldrighi JM, Ramires JAF.
Tendência do risco de morte por doenças circulatórias, cerebrovasculares e isquêmicas do coração em treze estados do Brasil, de 1980 a 1998. Arq Bras Cardiol. 2006; 87: 641-8.

21. Lessa I. Epidemiologia das doenças crônicas não-transmissíveis. São Paulo, Rio de Janeiro: HUCITEC, 1998. p. 97-114.

22. Souza MFM, Alencar APA, Malta DC, Moura L, Mansur AP. Análise de séries temporais da mortalidade por doenças isquêmicas do coração e cerebrovasculares, nas cinco regiões do Brasil, no período de 1981 a 2001. Arq Bras Cardiol. 2006; 87 (6): 735- 40.

23. Godoy MF, Lucena JM, Miquelin AR, Paiva FF, Oliveira DLQ, Augustin Junior IL, et al. Mortalidade por doenças cardiovasculares e níveis socioeconômicos na população de São José do Rio Preto, Estado de São Paulo, Brasil. Arq Bras Cardiol. 2007; 88 (2): 200-6.

24. Daniel E, Germiniani H, Nazareno ER, Braga SV, Winkler AM, Cunha CP. Tendência de mortalidade por doenças isquêmicas do coração na cidade de Curitiba - Brasil, de 1980 a 1998. Arq Bras Cardiol. 2005; 85(2): 100-4.

25. Sousa LB, Souza RKT, Scochi MJ. Hipertensão arterial e saúde da família: atenção aos portadores em município de pequeno porte na região sul do Brasil. Arq Bras Cardiol. 2006; 87: 496-503.

26. Abreu DMX, César CC, França EB. Relação entre as causas de morte evitávei por atenção à saúde e a implementação do Sistema Único de Saúde no Brasil. Rev Panam Salud Publica. 2007; 21 (5): 282-91.

27. Collins R, Peto R, MacMahon S, Hebert P, Fiebach NH, Eberlein KA, et al. Blood pressure, stroke, and coronary heart disease. Part 2: Short-term reductions in blood pressure: overview of randomized drug trials in their epidemiological context. Lancet. 1990; 335: 827-38.

28. Lotufo PA. Epidemiologia das doenças isquêmicas do coração no Brasil. In Lessa I. Epidemiologia das doenças crônicas não-transmissíveis. São Paulo Rio de Janeiro: HUCITEC; 1998. p. 115-22.

29. Oliveira GMM, Klein CH, Silva NAS, Godoy PH, Fonseca TMP. Letalidade por doenças isquêmicas do coração no estado do Rio de Janeiro no período de 1999 a 2003. Arq Bras Cardiol. 2006; 86 (2):131-7.

30. Rosengren A, Hawken S, Ôunpuu S, Sliwa K, Zubaid M, Almahmeed WA et al. Association of psychosocial risk factors with risk of acute myocardial infarction in 11119 cases and 13648 controls from 52 countries (the INTERHEART study): case-control study. Lancet. 2004; 364: 953-62.

31. Polanczyk CA. Fatores de risco cardiovascular no Brasil: os próximos 50 anos! Arq Bras Cardiol. 2005; 84(3): 199-201.

32. Ministério da Saúde. Política Nacional de Promoção da Saúde. Brasília 2002.

33. Ministério da Saúde. Portaria GM no 154, de 24 de Janeiro de 2008. Cria os Núcleos de apoio à Saúde da Família - NASF. Diário Oficial da União 4 mar 2008; Seção 1(43):38-42. 